\title{
Pulmonary and Cerebral Infarcts Due to Secondary Thrombosis Risk of a Genetic Mutation: Life-threating Methylentetrahydrofolate Reductase (MTHFR) Deficiency with Early Onset
}

\author{
lanosi Edith Simona*, Szasz Simona, Nemes Roxana, Jimborean Gabriela
}

University of Medicine and Pharmacy, Targu Mures, Romania

Methylentetrahydrofolate reductase (MTHFR) is a key enzymatic component of the folate cycle, converting 5,10-methylentetrahydrofolate into 5-methylentetrahydrofolate. Severe MTHFR deficiency is a rare recessive disease leading to major hyperhomocysteinemia, homocystinuria, and progressive neurological distress within the two first decades of life.

We present the case of a young, 21 years old female patient who was admitted and treated in Clinic of Pneumology Tirgu Mures for a posterobasal left pneumonia without favourable radiologic evolution under antibiotic and symptomatic treatment. Thoracotomy was recommended in order to elucidate the diagnosis. The histopatological examination revealed the zone of pulmonary infarction. After 12 weeks from surgical intervention, the patient was admitted in Department of Neurology for stroke attack. The complex laboratory investigations reveal deficiency of methylentrahydrofolate reductase (MTHFR) caused by a genetic mutation.

Keywords: pulmonary and cerebral infarcts, genetic mutation, MTHFR deficiency

Received: 28 December 2015 / Accepted: 20 March 2016

\section{Introduction}

The most common causes of morbidity and mortality in our societies is represented by the thrombosis. Haemostatic disequilibrium is the key mechanism at the origin of all type of thrombosis. The pathogenesis of venous thromboembolism, a complex and multifactorial process, involves the interaction of acquired factors such as ageing, obesity, prolonged immobilization (bed rest, long travel and fracture), surgery, pregnancy and post-partum, and oral contraceptives or cancer, with a series of genetic predisposing conditions. Recent studies revealed several alterations in the genetics of haemostatic factors increasing the risk of thrombosis. MTHFR deficiency, a rare autosomal recessive condition, belongs to the homocysteine remethylation disorders which also include defects in cytoplasmic cobalamin processing, and functional MTR deficiencies $[1,2]$. In MTHFR-deficient patients, the onset of clinical symptoms mainly occurs during the two first decades of life [3-5]. More than 50 private loss-of-function mutations have been reported in severe MTHFR-deficient patients. A majority of these patients are compound heterozygotes, while homozygotes are described occasionally, often in consanguineous families or in particular ethnic groups [6].

\section{Case presentation}

We present the case of a young, 21 years old female patient, hospitalized in the Pneumology Clinic Tg.Mures, between 01.07 2009-28.07 2009.

* Correspondence to: Edith Simona lanosi

E-mail: ianosi_edith70@yahoo.com
The patient was referred to the Pneumology Clinic after previously being treated in the Medical Clinic for 10 days for a left basal pleuro-pneumonia with unfavourable evolution during the unspecific antibiotherapy and symptomatic treatment. The patient was sent to our clinic with suspicion of pleural tuberculosis.

The anamnesis and medical examination at the admission in our hospital evidences no major personal pathologic antecedents, pain in left hemithorax which worsened in deep inspiration and cough. We mention the use of oral contraception before hospitalization.

Clinically, she presented relatively good general health status, pale skin and diminished vesicular murmurs at the left pulmonary base.

Biochemistry parametersindicated: ESR: 90/124, L: 6800, Hgb: $11.4 \mathrm{mg} / \mathrm{dl}$, Hct: 34.8\%, Glucose: $92 \mathrm{mg} / \mathrm{dl}$, Urea: $21.5 \mathrm{mg} / \mathrm{dl}$, Creatinine: $0.77 \mathrm{mg} / \mathrm{dl}$, ALP: $10 \mathrm{U} / \mathrm{L}$, ALT: 27 U/l, Rheumatoid factor: Negative, ANCA: Negative, anti-dsDNA:Negative, CIC:65 U/l. Bacteriological examination was negative for nonspecific flora. Intrademoreaction with 2UI PPD was $0 \mathrm{~mm}$ and sputum examination for BK was negative at microscopy/sterile in culture. The chest X-rays (front and profile) showed an opacity localised postero-basale in the left inferior lobe (Figure 1).

Treatment with antibiotics, antimicotics was initiated, associated with symptomatic therapy, consisting in: Clind amycin+Ciprofloxacin+Gentamicin, Ketoprofen, Fluconazol, Codeine and Paracetamol. Left pleural punction was white. During the treatment (1 July 2009-15 July 2009) the patient became afebrile, with good clinical and biological status but with persistent left chest pain and without 


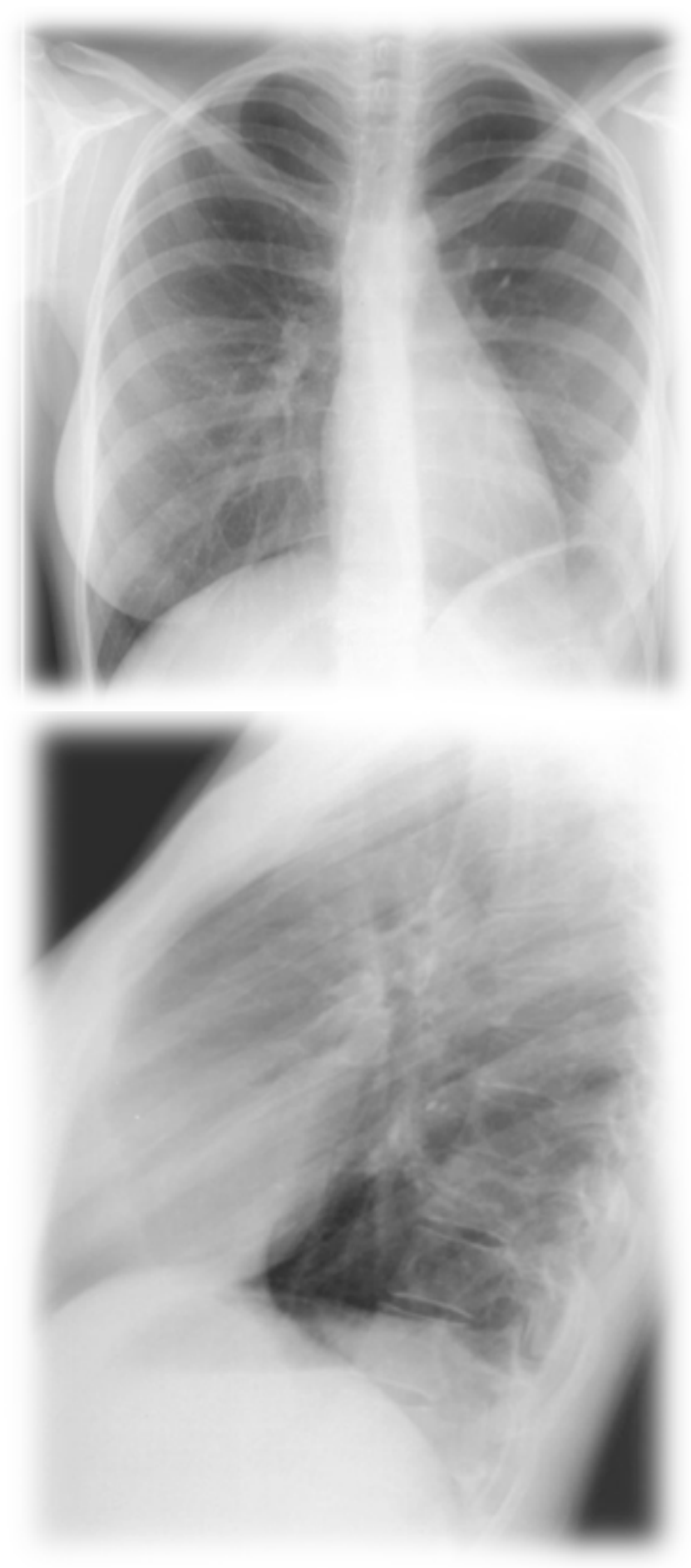

Fig.1. Postero-basal left sided pneumonia

favourable evolution on chest X-ray, therefore a thoracic computer tomography was performed.

Chest CT revealed a pseudonodulary lesion with mixt density at the site of the left basal lung, which was solide, liquidy and airy tangent to the laterothoracic pleura. CT examination concluded that the lesion was a benign pneumonia with incipient necrosis or abscess. After The suspision of abscess indicated the initiation of unspecific antibiotic therapy, continued with: Timentin, Metronidazol, Ciprofloxacin, Fluconazol, Codeine, Ketoprofen, C Vitamine for 2 weeks.
The biochemistry tests have been repeated, indicated ESR value of 80/102, while all the other values were normal.

After 2 weeks of treatment the chest CT was repeated, indicating a solid mass, irregular and triangulare in the VIII segment of the posterior left basale lobe, close to the oblical scisurae. Many vascular structures were evidentiated between this mass and hilus, indicating a structure of unknown etiology (pleuropulmonary tumor?, chronicized pneumonia?) (Figure 2). Thoracotomy with biopsy and histopatological examination were recommended at discharge.

The patient was hospitalized at the Thoracic Surgery Department in Cluj-Napoca between 04.08.2009 - 20.08. 2009.The surgical intervention was made on 11.08. 2009, consisting in an atypical excision of the pulmonary lesion. Histopathological examination revealed an area of pulmonary infarction [7], representing a chronic pulmonary inflammatory process associated with obliterant bronchiolitis and thrombotic aspects with the recommendation to exclude an associated cardiac pathology or an antyphospholipidic syndrome. The patient refused any further investigations and at 11.11. 2009 presented again at the Pneumology Clinics from Targu-Mures with strong headache, nausea, vomiting and speech disorders.

Urgent cranial CT and neurological examination were recommended and cranial CT indicated a $9 \mathrm{~mm}$ nodule spontaneously hyperdense and intense iodophile situated in the projection area of the lateral sigmoid sinus, raising the suspicion of left posterior sylvian ischemic AVCI, caused by a vasculary malformation or aneurysm (Figure 3 ).

Chest CT showed many hyperdense strips with rectractile character on the left posterobasal lobe.

The patient was hospitalized urgently at the Neurology Department. Angio MRI was highly suggestive for a secondary ischemic cerebral vasculary attack of a cerebral thrombophlebitis, evidentiating the obstruction of the left transverse sinus and the superior saggital sinus $[6,8,9]$. Biochemistry and immunological examinations established a mutation of MTHFR C677T, which is a heterozygote genetic risk of thrombosis.

Corroborating the clinico-paraclinic aspects, the diagnosis established was: cerebral thrombophlebitis of the left transverse sinus with left posterior sylvian ischemic AVC, resulting in crises of speech disorder, caused by a genetic heterozygote mutation of MTHFR C677T [1-3].

During the hospitalization the patient was treated with anticoagulants (Sintrom), anticonvulsives, antibiotics, cerebral depletives with favourable evolution. She leaves hospital after 11 days with the recommendation of continuing the anticoagulant, anticonvulsive treatment, periodical neurological screening, interdiction of use oral contraceptives, banned of alcohol, coffee use, no physical or psychical effort.

The patient was reevaluated after 12 weeks, when she reproted that she was feeling well, following the treatment 


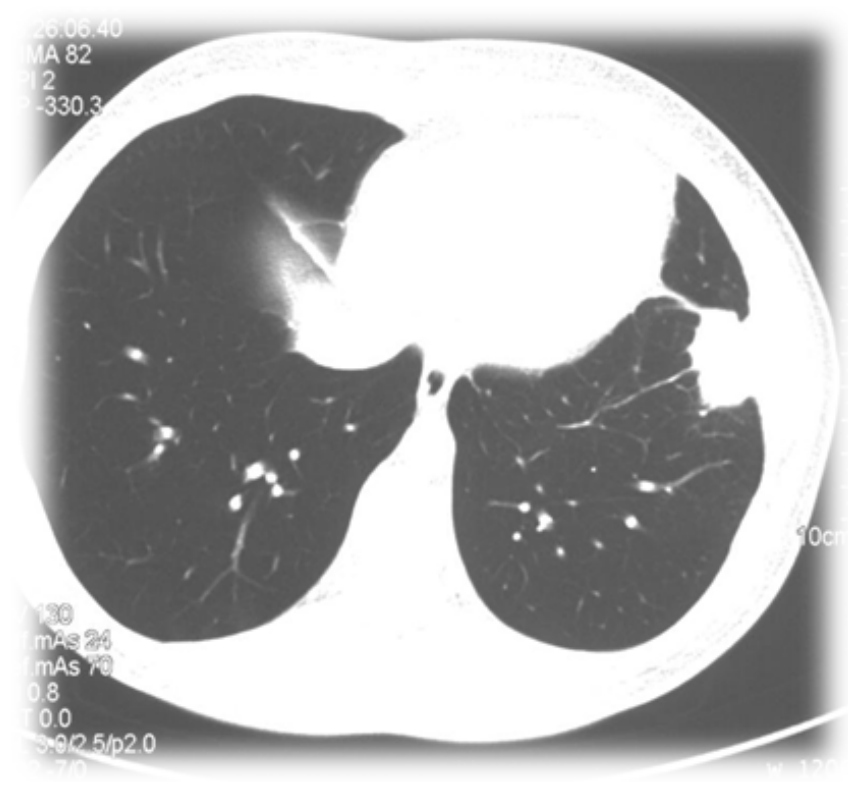

Fig. 2. Chest CT indicating indicating a solid mass, irregular and triangulare in the VIII segment of the posterior left basale lobe

with Sintrom and Carbamazepin. Repeated pulmonary $\mathrm{X}$-ray did not evidentiate nay modifications as compared with the previous X-ray.

\section{Discussions}

We present the case of a young female patient whith pulmonary and cerebral infarcts at a very early age -21 , without any special thrombotic conditions before admission at the hospital. This situation recommend always a complex genetic investigation [3,5].

The appearance of these thrombosis could be favorized by the use of oral contraceptives and infectious episodes. Mutation of MTHFR gene and deficiency of B6, B1 2 vitamins are associated with the increasing seric concentration of homocysteine $[3,4,10]$, and hyperhomocysteinemia is an important risk factor of atherosclerosis and thrombosis. Hyperhomocysteinemia fastens the vasculary endothelial cellular dysfunctions also in the process of atherosclerosis by increasing nitric oxide in vascular muscle cell levels. In this case association of the highly genetic risk of thrombosis due to the heterozygote mutation of the methilentetrahydropholatreductasis (MTHFR) gene was the cause of infarcts [7].

\section{Conclusions}

In conclusions in case of apperance thrombosis at early age it is highly recommended to perform complex genetic tests. In these cases thrombotic complications should be avoided by using chronic use of oral anticoagulants or fractionated heparine and by avoiding other conditions which favorize the thrombogenesis, such as: infections, traumas, contraceptive treatment or smoking.

Further studies about the genetic,medical and nutritional factors affecting the MTHFR polymorphism, as well as

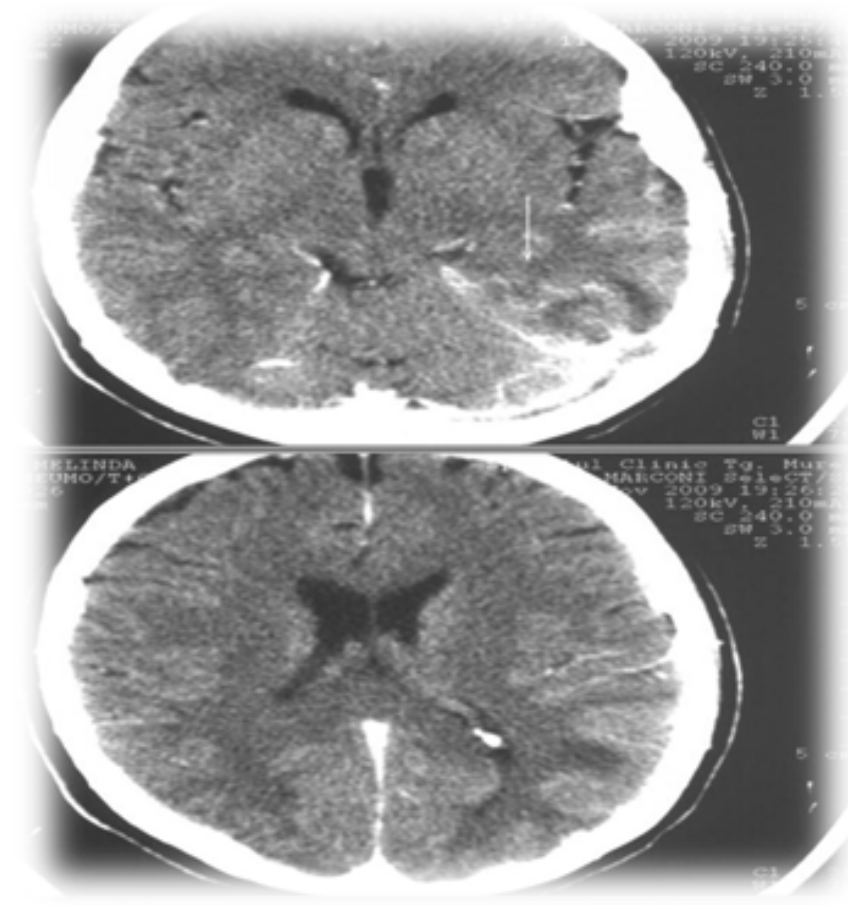

Fig. 3. Cranial CT indicating a hyperdense $9 \mathrm{~mm}$ nodule

a better understanding of human demographic history, is needed for implementing novel diagnostic and therapeutic options into clinical practice.

\section{Conflicts of interest}

The authors report no conflicts of interest.

\section{References}

1. Forges T, Chery C, Audonnet $S$, et al. Life-threatening methylenetetrahydrofolate reductase (MTHFR) deficiency with extremely early onset: Characterization of two novel mutations in compound heterozygous patients.Molecular Genetics and Metabolism 2010;100:143-148;

2. K. Kchock, A. Omezzine, L. Rebhi, et al. Preanalytical conditions for the measurement of plasma total homocysteine: genotype C677T MTHFR dependent non-fasting variations and anticoagulant effects on stability. Immuno-analyse et biologie spécialisée 2007;22 :319-324;

3. Nazki FH, Sameer AS, Ganaie BA. Folate: Metabolism, genes, polymorphisms and the associated diseases. Gene. 2014;533:11-20;

4. Simsek E, Yesilyurt A, Pinarli F, Eyerci N, Ulus AT. Combined genetic mutations have remarkable effect on deep venous thrombosis and/or pulmonary embolism occurence: Gene. 2014;536:171-176;

5. Joachim E, Goldenberg NA, Bernard TJ, etal. The Methylentetrahydrofolate Reductase Polymorphism (MTHFR c.677C > T) and Elevated Plasma Homocysteine Levels in a U.S.Pediatric Population with Incident Thromboembolism. Thromb Res. 2013;132:170-174;

6. Del Balzo F, Spalice A, Perla M, Properzi E, lannetti P. MTHFR homozygous mutation and additional risk factors for cerebral infarction in a large italian family. Pediatr Neurol. 2009;40:63-67;

7. Cha SI1, Shin KM, Lee J, et al. Clinical relevance of pulmonary infarction in patients with pulmonary embolism. Thromb Res. 2012;130:e1-e5;

8. Li P, Qin C. Methylenetetrahydrofolate reductase (MTHFR) gene polymorphisms and susceptibility to ischemic stroke: A meta-analysis. Gene. 2014;535:359-364;

9. Sazci A, Ergul E, Tuncer N, Akpinar G, Kara I. Methylenetetrahydrofolate reductase gene polymorphisms are associated with ischemic and hemorrhagic stroke: Dual effect of MTHFR polymorphisms C677T and A1298C. Brain Res Bull. 2006;71:45-50;

10. Gouveia LO, Canhão P. MTHFR and the risk for cerebral venous thrombosis- a meta-analysis. Thromb Res. 2010;125:e153-e158; 